University of Nebraska - Lincoln

DigitalCommons@University of Nebraska - Lincoln

April 1982

\title{
Random-phase approximation to one-body transition matrix elements for open-shell atoms
}

Anthony F. Starace

University of Nebraska-Lincoln, astarace1@unl.edu

Siamak Shahabi

University of Nebraska - Lincoln

Follow this and additional works at: https://digitalcommons.unl.edu/physicsstarace

Part of the Physics Commons

Starace, Anthony F. and Shahabi, Siamak , "Random-phase approximation to one-body transition matrix elements for open-shell atoms" (1982). Anthony F. Starace Publications. 22.

https://digitalcommons.unl.edu/physicsstarace/22

This Article is brought to you for free and open access by the Research Papers in Physics and Astronomy at DigitalCommons@University of Nebraska - Lincoln. It has been accepted for inclusion in Anthony F. Starace Publications by an authorized administrator of DigitalCommons@University of Nebraska - Lincoln. 


\title{
Random-phase approximation to one-body transition matrix elements for open-shell atoms
}

\author{
Anthony F. Starace \\ Behlen Laboratory of Physics, The University of Nebraska, Lincoln, Nebraska 68588* \\ and Fakultät für Physik der Universität Freiburg, D-7800 Freiburg, \\ Federal Republic of Germany \\ Siamak Shahabi \\ Behlen Laboratory of Physics, The University of Nebraska, Lincoln, Nebraska 68588
}

(Received 27 April 1981)

\begin{abstract}
A graphical procedure is presented for evaluating each term in the equation satisfied by the first-order transition matrix for an arbitrary (open-shell) atom. For those terms involving the interaction of excited virtual pairs of electrons with the ionic core, we introduce the approximation that there is no exchange of orbital or spin angular momentum with the ionic core. This approximation is shown to lead to the random-phase approximation (RPA) equations in the closed-shell-atom case; we use it to define the RPA for openshell atoms. The single equation satisfied by the first-order transition matrix is used to obtain a set of $N+N^{\prime}$ coupled differential equations for $N$ final-state radial functions and $N^{\prime}$ initial-state radial functions, which together, completely determine the first-order transition matrix for an atomic system having $N$ final-state channels. (The relation of $N^{\prime}$ to $N$ depends on the particular atom studied.) The $N+N^{\prime}$ differential equations are shown to reduce to familiar forms in the following cases: (1) When initial-state correlations are ignored, one obtains the close-coupling equations and (2) when closed-shell atoms are considered, $N=N^{\prime}$ and one obtains the $2 N$ coupled differential equations of the Chang-Fano version of the RPA. Finally, our RPA first-order transition matrix is used to evaluate the matrix element of the electric-dipole operator for an arbitrary (open-shell) atom. These RPA electric-dipole transition matrix elements may be used to calculate nonrelativistically all experimentally observable quantities resulting from a single-electron atomic photoabsorption process.
\end{abstract}

\section{INTRODUCTION}

Theoretical understanding of photoionization cross sections for closed-shell atoms is currently based on the random-phase approximation (RPA). ${ }^{1-3}$ In addition to the usual final-state interactions that are treated by Hartree-Fock or close-coupling calculations, the RPA also treats the effects due to excitations of virtual pairs of valence electrons in both the initial state and the final state. A major result of such virtual excitations is the reduction of the effective strength of final-state interactions. Experience has shown that the particular electron correlations included in the RPA for closed-shell atoms are necessary to obtain good agreement of theoretical calculations with experimental data. In particular, the importance of these electron correlations has been confirmed for closed-shell atoms by other theoretical methods, such as the many-body perturbation theory, ${ }^{4}$ the R-matrix theory, 5,6 and the multiconfiguration Hartree-Fock theory. ${ }^{7}$ Theoretical understanding of the influence of electron correlations on the photoionization cross sections for open-shell atoms, on the other hand, is less developed. This is due to the greater theoretical difficulty of dealing with atoms which are not spherically symmetric and which thus have a greater number of final-state channels. While RPA theories have been developed to treat open-shell atoms, ${ }^{8-12}$ these have been given in the form of matrix or integral equations which require the use of large numbers of basis functions for their solution.

In this paper we define a random-phase approximation for transition matrix elements in a way that is independent of whether one is dealing with a 
closed- or an open-shell atom. We begin by calculating the first-order transition matrix ${ }^{13,14}$ for a general (open- or closed-shell) atom using a graphical method. This transition matrix may be used to calculate matrix elements of a general one-body operator having rank $\lambda$ in orbital space and $\sigma$ in spin space. In particular, it may be used to calculate matrix elements of the electric-dipole operator (having $\lambda=1$ and $\sigma=0$ ), from which one may obtain the photoionization cross section as well as other experimentally measurable quantities related to photoabsorption processes. The graphical evaluation of the first-order transition matrix greatly simplifies the treatment of antisymmetrization and of angular-momentum algebra and in addition affords an insight into the physical processes involved that is similar to that afforded by the diagrams of ordinary many-body perturbation theory.

In evaluating the first-order transition matrix we follow Chang and Fano ${ }^{14}$ in assuming that the ground state may contain pairs of virtually excited orbitals. While our graphical procedure allows us to evaluate the interactions of the virtually excited orbitals with the residual ion, such interactions are exceedingly complex. These particular interaction diagrams are evaluated approximately here. We assume, firstly, that when the virtually excited electrons interact with the residual ion there is no exchange of orbital or spin angular momentum, and, secondly, that the Pauli principle is ignored. Since we find that these approximations give the ordinary RPA equations in the closed-shell case, we define our RPA by these approximations whether we are dealing with either an open- or a closedshell atom.

As a result of our RPA we find that the firstorder transition matrix for an atom having $N$ final-state channels is determined by a set of $N$ final-state radial functions and $N^{\prime}$ initial-state radial functions which satisfy $N+N^{\prime}$ coupled differential equations. (The relation of $N^{\prime}$ to $N$ depends on the particular atom studied.) Large numbers of basis functions are thus not needed in a calculation by this method. The $\left(N+N^{\prime}\right)$ coupled differential equations reduce to familiar forms in the following limiting cases: (1) When initial-state correlations are ignored we obtain the $N$ coupled differential equations of the close-coupling approximation, ${ }^{15}$ (2) When the atom has only closed shells, $N=N^{\prime}$, and we obtain the $2 N$ coupled differential equations of the Chang-Fano version of the RPA. ${ }^{14,16}$

In Sec. II we review the properties of the transition matrix. In Sec. III we describe our graphical method for evaluating the various terms in the equation satisfied by the transition matrix and introduce our random-phase approximation. In Sec. IV we obtain $\left(N+N^{\prime}\right)$ coupled differential equations for the $\left(N+N^{\prime}\right)$ radial functions which define the transition matrix. These equations are shown to reduce to familiar forms in special cases. Finally, in Sec. V we calculate the RPA form for the electric-dipole reduced matrix element. Preliminary reports of our graphical method ${ }^{17}$ and of our definition of the random-phase approximation ${ }^{18}$ have been given elsewhere.

\section{THE FIRST-ORDER TRANSITION MATRIX}

We assume that in a certain transition an $N$ electron atom may be described by an initial state $\langle i|$ and a final state $|f\rangle$. The first-order transition matrix for this transition, $\left\langle\overrightarrow{\mathrm{r}}_{N}^{\prime}|\hat{\Gamma}| \overrightarrow{\mathrm{r}}_{N}\right\rangle$, is defined as ${ }^{13,14}$

$$
\begin{aligned}
\left\langle\overrightarrow{\mathrm{r}}_{N}^{\prime}|\hat{\Gamma}| \overrightarrow{\mathrm{r}}_{N}\right\rangle \equiv N \prod_{j=1}^{N-1} \iint & d \overrightarrow{\mathrm{r}}_{j}^{\prime} d \overrightarrow{\mathrm{r}}_{j}\left\langle\overrightarrow{\mathrm{r}}_{1}^{\prime} \cdots \overrightarrow{\mathrm{r}}_{N}^{\prime} \mid f\right\rangle \\
& \times\left\langle i \mid \overrightarrow{\mathrm{r}}_{1} \cdots \overrightarrow{\mathrm{r}}_{N}\right\rangle \\
& \times \delta\left(\overrightarrow{\mathrm{r}}_{j}^{\prime}-\overrightarrow{\mathrm{r}}_{j}\right)
\end{aligned}
$$

For simplicity of notation, each $\vec{r}_{j}(1 \leq j \leq N)$ includes also the spin coordinate of the corresponding electron. The matrix element of any one-body operator $\hat{O}^{\lambda, \sigma}(\overrightarrow{\mathrm{r}})$ of rank $\lambda$ in orbital space and rank $\sigma$ in spin space may be determined from the first-order transition matrix by means of the following one-electron integral:

$$
\begin{aligned}
\left\langle i\left|\hat{O}^{\lambda, \sigma}\right| f\right\rangle=\int d \overrightarrow{\mathrm{r}}_{N}^{\prime} \int & d \overrightarrow{\mathrm{r}}_{N}\left\langle\overrightarrow{\mathrm{r}}_{N}^{\prime}|\hat{\Gamma}| \overrightarrow{\mathrm{r}}_{N}\right\rangle \\
& \times \hat{O}^{\lambda, \sigma}\left(\overrightarrow{\mathrm{r}}_{N}\right) \delta\left(\overrightarrow{\mathrm{r}}_{N}^{\prime}-\overrightarrow{\mathrm{r}}_{N}\right) .
\end{aligned}
$$

The forms of the final-state wave function $\left\langle\vec{r}_{1}^{\prime} \cdots \vec{r}_{N}^{\prime} \mid f\right\rangle$ and the initial-state wave function $\left\langle i \mid \overrightarrow{\mathrm{r}}_{1} \cdots \overrightarrow{\mathrm{r}}_{N}\right\rangle$ as well as the one-electron orbitals of which they are comprised must be specified in order to determine the first-order transition matrix and thereby, the desired matrix elements in Eq. (2). The forms of the initial and final states that we assume in this paper are specified below. First, though, we note that for any states $|i\rangle$ and $|f\rangle$ the outer product $|f\rangle\langle i|$ must satisfy the following dynamical equation if the atom is described by 
the Hamiltonian $H$ :

$$
\hbar \omega|f\rangle\langle i|=H| f\rangle\langle i|-| f\rangle\langle i| H .
$$

Here $\hbar \omega$ is the transition energy, $E_{f}-E_{i}$. If Eq.
(3) is integrated over $N-1$ coordinates then on the left-hand side we obtain $\hbar \omega\left\langle\overrightarrow{\mathrm{r}}_{N}^{\prime}|\hat{\Gamma}| \overrightarrow{\mathrm{r}}_{N}\right\rangle$, while on the right we obtain partially integrated matrix elements of $H$ between the initial and final states:

$$
\begin{aligned}
\hbar \omega\left\langle\overrightarrow{\mathrm{r}}_{N}^{\prime}|\hat{\Gamma}| \overrightarrow{\mathrm{r}}_{N}\right\rangle= & N \prod_{j=1}^{N-1} \iint d \overrightarrow{\mathrm{r}}_{j}^{\prime} d \overrightarrow{\mathrm{r}}_{j}\left\langle\overrightarrow{\mathrm{r}}_{1}^{\prime} \cdots \overrightarrow{\mathrm{r}}_{N}^{\prime}|H| f\right\rangle\left\langle i \mid \overrightarrow{\mathrm{r}}_{1} \cdots \overrightarrow{\mathrm{r}}_{N}\right\rangle \\
& -N \prod_{j=1}^{N-1} \iint d \overrightarrow{\mathrm{r}}_{j}^{\prime} d \overrightarrow{\mathrm{r}}_{j}\left\langle\overrightarrow{\mathrm{r}}_{1}^{\prime} \cdots \overrightarrow{\mathrm{r}}_{N}^{\prime} \mid f\right\rangle\left\langle i|H| \overrightarrow{\mathrm{r}}_{1} \cdots \overrightarrow{\mathrm{r}}_{N}\right\rangle .
\end{aligned}
$$

For any particular form of the states $|f\rangle$ and $\langle i|$ in terms of one-electron orbitals, Eq. (4) represents a consistency equation for these orbitals which must be satisfied.

We consider in this paper one-electron transitions from the open subshell $n_{0} l_{0}^{q}$ of an atom having any number of other, closed subshells. We assume that there are $q$ electrons in the open subshell, where $2 \leq q \leq 4 l_{0}+2$. The closed-shell-atom case, $q=4 l_{0}+2$, is thus treated on the same footing as the open-shellatom case. Note that in the single-subshell approximation considered here the special case $q=1$ is trivial and is not treated explicitly. On the other hand, going beyond the single-subshell approximation (by considering excitations out of one or more of the closed subshells as well as out of the open subshell $n_{0} l_{0}^{q}$ ) represents a complicated but nevertheless straightforward generalization of the theory presented here. For simplicity of notation, therefore, we do not consider such a general case in this paper.

For the class of excitations to be considered in this paper, then we assume the $\alpha$ th final state to have the form

$$
\left|f^{\alpha}\right\rangle \equiv \sum_{\tilde{L}^{\prime} \tilde{S}^{\prime} l^{\prime}}\left|n_{0} l_{0}^{q-1}\left(\tilde{L}^{\prime} \widetilde{S}^{\prime}\right) \psi_{\left(\tilde{L}^{\prime} \tilde{S}^{\prime}\right) \epsilon l^{\prime}}^{\alpha} L_{f} S_{f}\right\rangle
$$

Here $\alpha$ specifies a particular set of final-state quantum numbers, $\psi_{\left(L^{\prime} S^{\prime}\right) \epsilon l^{\prime}}^{\alpha}(r)$ is an unknown radial wave function for the excited electron, and the ionic core wave functions are assumed to be neutral atom HartreeFock wave functions. ${ }^{19}$ The initial state is assumed to have the following correlated form:

$$
\langle i| \equiv\left\langle n_{0} l_{0}^{q} L_{i} S_{i}\right|+\sum_{L_{p} S_{p}} \sum_{\bar{L} \bar{S} l_{\phi}} b\left(\bar{L} \bar{S}, L_{p} S_{p}, l_{\phi}\right)\left\langle n_{0} l_{0}^{q-2}(\bar{L} \bar{S}) \phi_{a} \phi_{b}\left(L_{p} S_{p}\right) L_{i} S_{i}\right|
$$

Here $\phi_{a}$ and $\phi_{b}$ are the radial wave functions for a pair of electrons excited out of the initial-state configuration, each having the orbital angular momentum $l_{\phi}$. (A more general form for $\langle i|$, not considered here, would permit $\phi_{a}$ and $\phi_{b}$ to have different orbital angular momenta.) The coefficients $b$ $\left(\bar{L} \bar{S}, L_{p} S_{p}, l_{\phi}\right)$ give the weighting of the correlation terms. The orbital wave functions of the configurations $n_{0} l_{0}^{q}$ and $n_{0} l_{0}^{q-2}$ are also taken to be the neutral atom Hartree-Fock orbital wave functions. ${ }^{19}$

The purpose of this paper is to present the coupled differential equations that one obtains (upon substituting Eqs. (5) and (6) in the dynamical equation (4) ) for the unknown final-state radial wave functions $\psi_{\left(\tilde{L}^{\prime} \tilde{S}^{\prime}\right) \in l^{\prime}}^{\alpha}(\boldsymbol{r})$ and certain linear combinations of the unknown initial-state radial wave functions $\phi_{a}(r)$ and $\phi_{b}(r)$. Once these unknown radial functions are determined, the transition matrix is completely defined by Eqs. (5) and (6) and the definition in Eq. (1). In the next section we present a graphical method for evaluating the integrals in Eq. (1) and on the right in Eq. (4).

\section{GRAPHICAL EVALUATION OF TERMS IN THE DYNAMICAL EQUATION}

\section{A. Generalities}

The dynamical equation, Eq. (4), relates the transition matrix to interaction matrix elements involving both the final state [first term on the right in Eq. (4)] and the initial state [second term on the right in Eq. (4)]. These matrix elements may be evaluated graphically. We have adapted for this purpose the graphical rules of Briggs $^{20}$ for 
representing state vectors and for evaluating matrix elements of one-body operators and of the electron correlation operator $\sum_{i>j} r_{i j}^{-1}$. Brigg's graphical rules, ${ }^{20}$ however, must be modified to accommodate our integrating over only $N-1$ electron coordinates in Eqs. (1) and (4) instead of all $N$.

The general idea of the graphical method is to represent an atomic state vector as the addition of the net orbital and spin angular momenta, represented graphically by lines, for each atomic subshell to form the total orbital and spin angular momenta of the state. The addition of each pair of angular momenta is affected analytically by Clebsch-Gordan coefficients, which are represented graphically by nodes having three lines attached, with one of the lines, having a bar attached, representing the resultant angular momentum. The angular-momentum properties of any operators which connect initial- and final-state vectors are also given a graphical representation. An algebraic expression for a given transition matrix element is then obtained by suitably joining the corresponding initial- and final-state vector graphs with the appropriate transition operator graph to form a standardized angular-momentum diagram. This procedure effects implicitly all necessary summations over magnetic quantum numbers as well as integration over angular and radial coordinates; the antisymmetry of the initial- and final-state vectors as well as the equivalence of electrons are taken into account by an appropriate multiplicative weighting factor. This resultant diagram may be reduced analytically to the product of a number, which is the desired angular factor, and a reduced radial matrix element.

\section{B. Notational rules}

Our graphical representation for the first-order transition matrix, defined analytically in Eq. (1), when the final and initial states are represented by Eqs. (5) and (6), is shown in Fig. 1. Except for the lack of integration over the $N$ th electron's coordinates, Fig. 1(a) represents the overlap of the final state (on the right) with the major configuration of the initial state (on the left). Figure 1(b) represents the overlap of the final state (on the right) with the doubly excited configurations contained in the ground state (on the left).

In what follows we shall use Fig. 1 to illustrate the basic graphical angular-momentum notational rules used in this paper. The reader is referred to the book by Jucys et al. $^{21}$ for a more general discussion of angular-momentum graphs and to the review by Briggs $^{20}$ for the graphical representation of state vectors and interaction operators. Each line in Fig. 1 represents both an orbital and a spin angular momentum. These angular momenta are added at each node to resultant orbital and spin angular momenta whose lines have a thick bar attached. Thus the node on the left in Fig. 1(a) represents the addition of the single-electron orbital angular momentum $l_{0}$ and, implicitly, the spin $\frac{1}{2}$ to the orbital and spin angular momenta $\widetilde{L}$ and $\widetilde{S}$ of the configuration $n_{0} l_{0}^{q-1}$ to obtain the resultant orbital and spin angular momenta $L_{i}$ and $S_{i}$ of the initial configuration $n_{0} l_{0}^{q}$. Note that in cases such as this where a single electron is split off from other equivalent electrons in the same subshell, a square node is used as a reminder that a coefficient of fractional parentage should multiply the angular factor represented by this diagram. The signs on each node indicate the order of addition of the angular momenta, + indicating a clockwise ordering and - indicating a counter-clockwise ordering. The phase convention chosen is that, when all orbitals are ordered downward with increasing energy (i.e., an electron from subshell $n_{1} l_{1}$ is written above one from subshell $n_{2} l_{2}$ if the orbital energies are such that $\left.\epsilon_{n_{1} l_{1}}<\epsilon_{n_{2} l_{2}}\right)$, then all nodes in the lefthand state vector diagram have a - sign and all nodes in the right-hand state vector diagram have $\mathrm{a}+$ sign. $^{20}$ Each of our diagrams will have initially four so-called "free" lines, which are lines at-

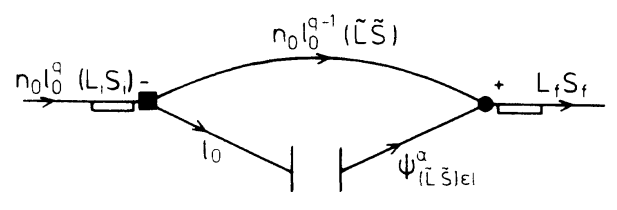

(a)

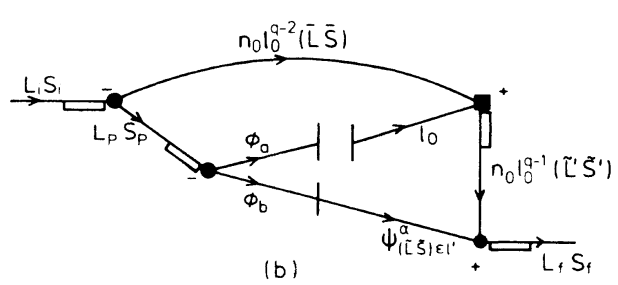

FIG. 1. Graphical representation of the first-order transition matrix where (a) indicates the overlap of the final state with the uncorrelated part of the initial state and (b) indicates the overlap of the final state with the correlated part of the initial state. 


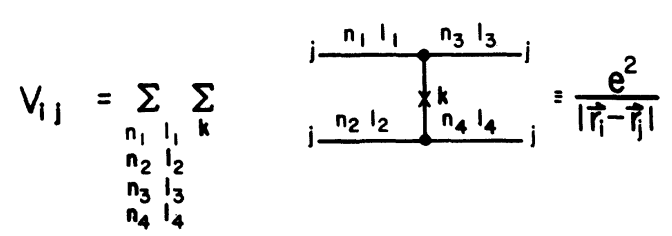

FIG. 2. Brigg's graphical representation of the Coulomb interaction operator.

tached to only a single node. Two of these represent the initial (i.e., $L_{i}$ and $S_{i}$ ) and final (i.e., $L_{f}$ and $S_{f}$ ) angular momenta and two represent the $N$ th electron orbital, in the initial and final configuration, whose coordinates are not integrated over. These four lines have well-defined orbital and spin magnetic quantum numbers whose signs are positive when the arrow is directed away from the associated node and negative when the arrow is directed toward the associated node. [Note that when the free ends of two lines having the same orbital and spin angular momentum are joinedtaking care that the arrows are aligned-one is implicitly performing a summation over magnetic quantum numbers and an integration over electron coordinates. The $\widetilde{L} \widetilde{S}$ line in Fig. 1(a) is such a "closed" line, i.e., one bounded at each end by a node.] Finally, the vertical bars on the ends of the lines for the $N$ th electron in the initial- and finalstate diagram represent the associated radial, angular, and spin wave functions for the $N$ th electron. The bar on the lowest line in Fig. 1(b) represents the overlap $\left(\phi_{b} \mid \psi_{(\tilde{L} \tilde{S}) \epsilon l^{\prime}}^{\alpha}\right)$ of the wave functions $\phi_{b}(\overrightarrow{\mathrm{r}})$ and $\psi_{(\tilde{L} \widetilde{S}) \epsilon l^{\prime}}^{\alpha}(\overrightarrow{\mathrm{r}})$.

\section{Coulomb interaction graphs}

Figure 1 represents graphically the first-order transition matrix appearing on the left in Eq. (4) for final and initial states of the forms given in Eqs. (5) and (6). The scalar one-body operators appearing in the Hamiltonian on the right of Eq. (4) have similar graphical representations. The twobody Coulomb operator in $H$ leads, however, to a number of final- and initial-state correlation diagrams. These are obtained by joining the graphical representation of the initial and final states in Eqs. (5) and (6) to Brigg's ${ }^{20}$ graphical representation for the Coulomb interaction operator, shown in Fig. 2. When coordinates $\vec{r}_{i}$ and $\vec{r}_{j}$ are both integrated over, the cross in Fig. 2 represents the following reduced matrix element:

$$
(-1)^{k} X\left(k ; n_{1} l_{1} n_{2} l_{2}, n_{3} l_{3} n_{4} l_{4}\right) \equiv(-1)^{k} R^{k}\left(n_{1} l_{1} n_{2} l_{2}, n_{3} l_{3} n_{4} l_{4}\right)\left(l_{1} \| C^{(k)}|| l_{3}\right)\left(l_{2} \| C^{(k)}|| l_{4}\right),
$$

where

$$
\left(l|| C^{(k)}|| l^{\prime}\right) \equiv(-1)^{l}[l]^{1 / 2}\left[l^{\prime}\right]^{1 / 2}\left(\begin{array}{lll}
l & k & l^{\prime} \\
0 & 0 & 0
\end{array}\right),
$$

and where $R^{k}$ is the Slater integral of order $k$,

$$
R^{k}\left(n_{1} l_{1} n_{2} l_{2}, n_{3} l_{3} n_{4} l_{4}\right) \equiv \int_{0}^{\infty} \int_{0}^{\infty} d r_{i} d r_{j} \frac{r_{<}^{k}}{r_{>}^{k+1}} P_{n_{1} l_{1}}\left(r_{i}\right) P_{n_{2} l_{2}}\left(r_{j}\right) P_{n_{3} l_{3}}\left(r_{i}\right) P_{n_{4} l_{4}}\left(r_{j}\right)
$$

In Figs. 3-6 we show the graphical representation for all Coulomb interactions involving the subshell $n_{0} l_{0}$ that occur on the right-hand side of Eq. (4). Figure (3) represents the direct and exchange interaction of the final-state excited electron, having the radial wave function $\psi_{\left(\widetilde{L}^{\prime} \widetilde{S}^{\prime}\right) \epsilon l^{\prime}}^{\alpha}$, with the subshell $n_{0} l_{0}^{q-1}\left(\widetilde{L} \widetilde{S}^{\prime}\right)$. The final state is overlapped with the uncorrelated part of the ground state [first term on the right in Eq. (6)]. Notice the gap which indicates that the $N$ th coordinate is not integrated over. The vertical bars indicate the wave functions of the electrons in the initial and final states having coordinate $\overrightarrow{\mathbf{r}}_{N}$. Because the $N$ th coordinate is not integrated over, the cross on the Coulomb interaction operator is taken to represent, instead of Eq. (7), the following reduced matrix element ${ }^{14}$ :

$$
(-1)^{k}\left(l_{2}\left\|V^{k}\left(n_{1} l_{1,} n_{3} l_{3} ; r_{N}\right)\right\| l_{4}\right) \equiv(-1)^{k} \int_{0}^{\infty} d r \frac{r_{<}^{k}}{r_{>}^{k+1}} P_{n_{1} l_{1}}(r) P_{n_{3} l_{3}}(r)\left(l_{1}\left\|C^{(k)}\right\| l_{3}\right)\left(l_{2}\left\|C^{(k)}\right\| l_{4}\right)
$$




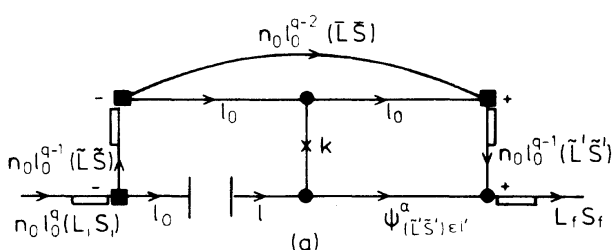

(a)

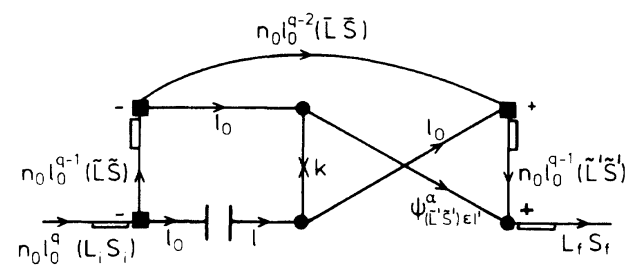

(b)

FIG. 3. Final-state direct (a) and exchange (b) interactions overlapped with the uncorrelated part of the initial state.

where $r_{<}=\min \left(r, r_{N}\right)$ and $r_{>}=\max \left(r, r_{N}\right)$. Thus, for example, in Fig. 3(a) the cross represents $(-1)^{k}\left(l|| V^{k}\left(n_{0} l_{0}, n_{0} l_{0} ; r_{N}\right)|| l^{\prime}\right)$ while in Fig. 3(b) the cross represents $(-1)^{k}\left(l|| V^{k}\left(n_{0} l_{0}, \psi_{\left(\tilde{L}^{\prime} \tilde{S}^{\prime}\right) \epsilon l^{\prime}}^{\alpha} ; r_{N}\right)|| l_{0}\right)$.

Similarly, Fig. 4 shows the graphical representation of final-state direct and exchange Coulomb interactions within the $n_{0} l_{0}$ subshell which are then
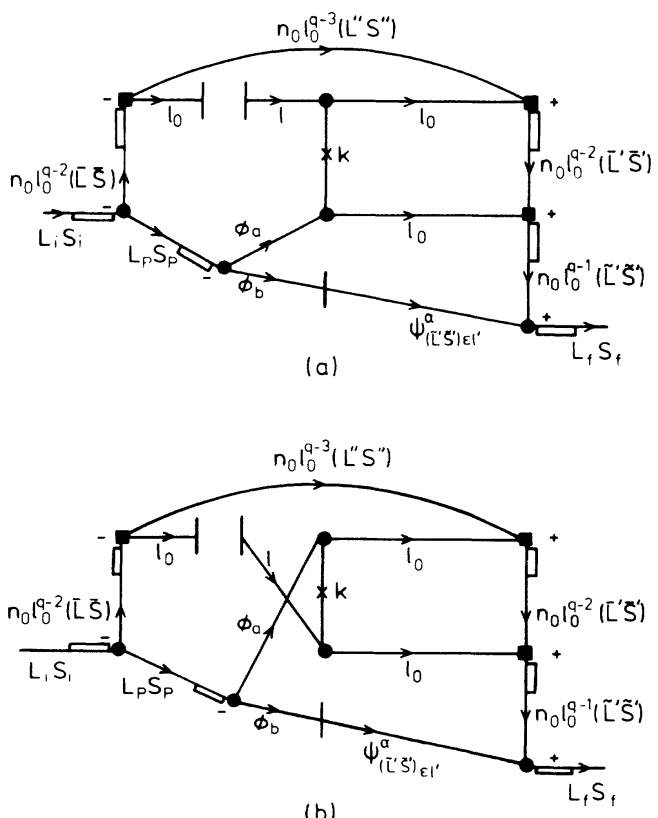

(b)

FIG. 4. Final-state direct (a) and exchange (b) interactions overlapped with the correlated part of the initial state.

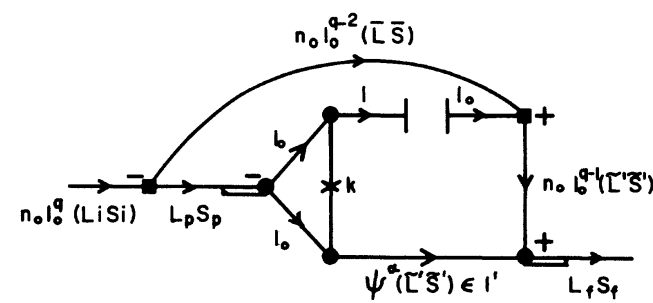

FIG. 5. Initial-state interaction, involving the uncorrelated part of the initial state, overlapped with the final state.
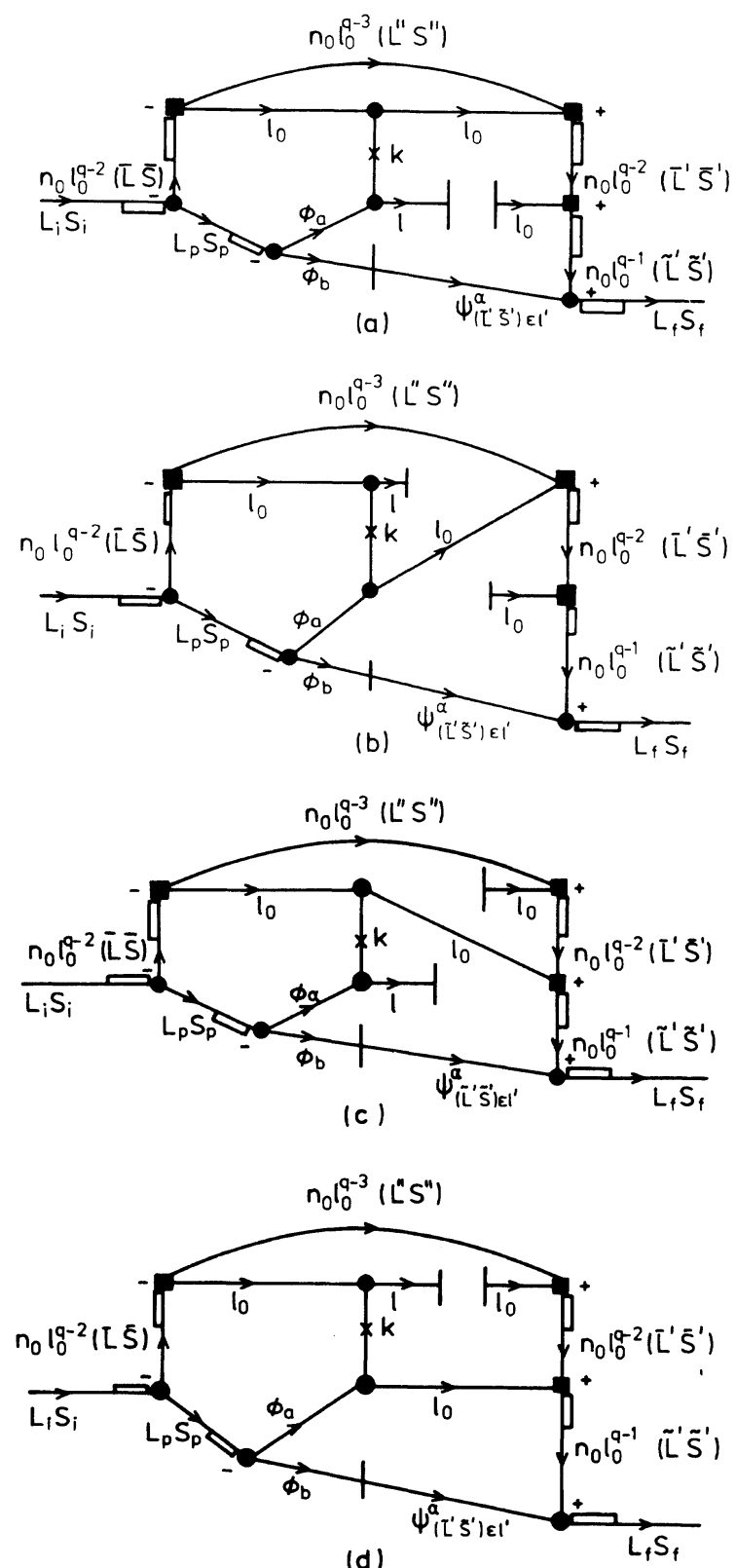

FIG. 6. Initial-state interactions, involving the correlated part of the initial state, overlapped with the final state. 
overlapped with the correlated part of the ground state [second term on the right in Eq. (6)]. Figure 5 shows direct and exchange Coulomb interactions within the uncorrelated part of the ground state which are then overlapped with the final state. Finally, Fig. 6 shows direct and exchange Coulomb interactions within the correlated part of the ground state. These involve the interactions of the functions $\phi_{a}$ and $\phi_{b}$ (only those involving $\phi_{a}$ are shown) with the $n_{0} l_{0}$ subshell. These correlated ground-state wave functions are then overlapped with the final-state wave function. Note that the diagrams corresponding to those in Fig. 6 in which $\phi_{a}$ and $\phi_{b}$ are interchanged differ only by the phase factor $(-1)^{L_{p}+S_{p}}$.
D. Removal of magnetic-quantum-number dependence

The magnetic-quantum-number dependence represented by the four free lines in each of the diagrams in Figs. 1, 3, 4, 5, and 6 may be factored out by a graphical procedure analogous to the WignerEckart theorem that results in diagrams with only closed lines. Figure 7 presents the details of this graphical procedure. In Fig. 7(a) are shown schematically the four free lines appearing in each of the diagrams in Figs. 1, 3, 4, 5, and 6. The orbital and spin angular momenta of the electron in the initial and final state with coordinate $r_{N}$ are expanded in Fig. 7(b) in terms of the sum $\lambda$ of the orbital angular momenta and the sum $\sigma$ of the spin

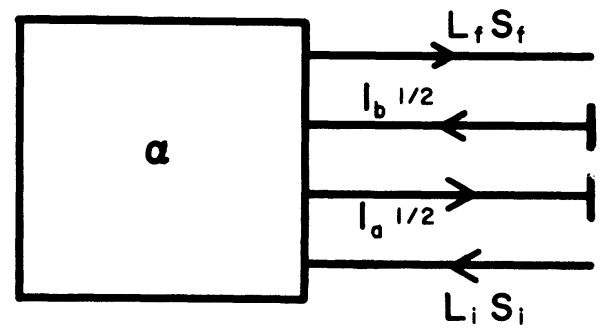

(a)

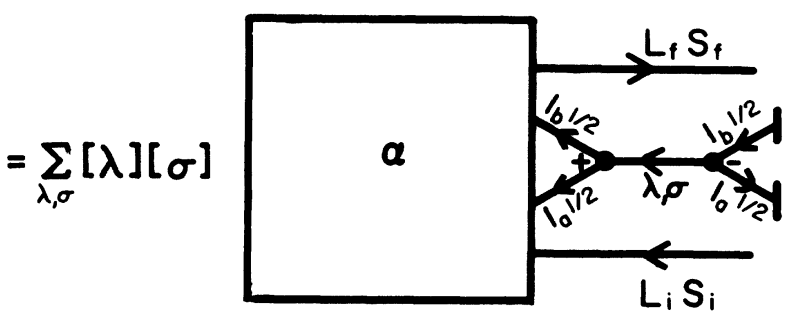

(b)

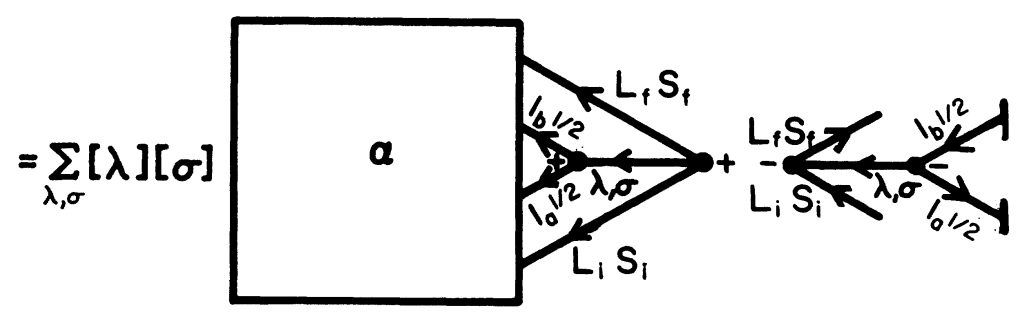

(c)

FIG. 7. Graphical procedure for separating out the magnetic-quantum-number dependence of the diagrams in Figs. $1,3,4,5$, and 6 . See discussion in text. 
angular momenta using the graphical "summation rule."22,23 In Fig. 7(c) the three parallel lines in Fig. 7(b) have been joined together and separated using the well-known graphical "rule of multiplication."22,23 The diagram with the four free lines on the right in Fig. 7(c) represents the algebraic expression given in Fig. 8, which summarizes our graphical rule for the removal of magnetic quantum number dependence from our diagrams. The double tensor operator $W_{m_{\lambda}, m_{\sigma}}^{\lambda, \sigma}$ appearing in Fig. 8 is defined as the following combination of angular and spin wave functions for the electron with coordinate $\overrightarrow{\mathrm{r}}_{N}$ (Ref. 14):

$$
\begin{aligned}
\left(l_{b}, \hat{r}_{b}\left|W_{m_{\lambda} m_{\sigma}}^{\lambda \sigma}\right| l_{a}, \hat{r}_{a}\right) \equiv \sum_{m_{a} m_{b}} Y_{l_{a} m_{a}}^{*}\left(\hat{r}_{a}\right) Y_{l_{b} m_{b}}\left(\hat{r}_{b}\right)(-1)^{l_{a}-m_{a}}\left(l_{b} m_{b}, l_{a}-m_{a} \mid \lambda m_{\lambda}\right) \\
\times \sum_{m_{a}^{1 / 2} m_{b}^{1 / 2}} \chi_{m_{a}^{1 / 2}}^{\dagger} \chi_{m_{b}^{1 / 2}}(-1)^{1 / 2-m_{a}^{1 / 2}}\left(\frac{1}{2} m_{b}^{1 / 2}, \frac{1}{2}-m_{a}^{1 / 2} \mid \sigma m_{\sigma}\right) .
\end{aligned}
$$

In Eq. (9) the $Y$ 's represent the spherical harmonics for the $N$ th electron in the initial and final states and the $\chi$ 's represent the corresponding two-component spin wave functions.

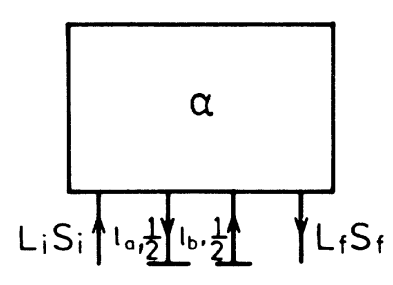

$$
\begin{gathered}
=\sum_{\lambda, \sigma} \sum_{m_{\lambda} m_{\sigma}}[\lambda]^{\frac{1}{2}}[\sigma]^{\frac{1}{2}} \\
X\left(\begin{array}{ccc}
L_{i} & \lambda & L_{f} \\
M_{i} m_{\lambda}-M_{f}
\end{array}\right)\left(\begin{array}{lll}
S_{i} \sigma & S_{f} \\
M_{S_{1}} m_{\sigma}-M_{S_{f}}
\end{array}\right)\left(l_{b}, \hat{r}_{b} \mid W_{m_{\lambda}, m_{\sigma}}^{\lambda, \sigma} l_{a}, \hat{r}_{a}\right) \Psi_{a}\left(r_{a}\right) \Psi_{b}\left(r_{b}\right)
\end{gathered}
$$

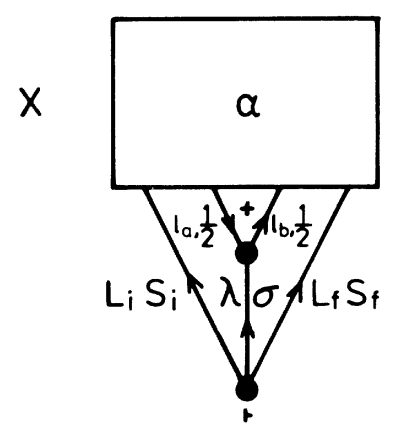

FIG. 8. Summary of graphical rule for separating out the magnetic-quantum-number dependence of the diagrams in Figs. 1, 3, 4, 5, and 6 . 


\section{E. The graphical RPA rule}

Our treatment has thus far been exact, given our choice of final and initial states in Eqs. (5) and (6) and our neglect of any virtual double excitations of the kind shown in Fig. 5 for any subshell other than $n_{0} l_{0}$. Those diagrams shown in Figs. 4 and 6 representing the interactions of the virtually excited orbitals $\phi_{a}$ and $\phi_{b}$ with the initial subshell $n_{0} l_{0}$, however, have very complex analytic expressions. The complexity stems from the presence of coefficients of fractional parentage which do not permit the analytic summation over the angular-momentum quantum numbers $L^{\prime \prime} S^{\prime \prime}$ of the configuration $n_{0} l_{0}^{q-3}$. These complex interactions occur also when $n_{0} l_{0}^{q}$ is initially a closed subshell with $q=4 l_{0}+2$. A simple approximation, however, permits the analytic summation over $L^{\prime \prime} S^{\prime \prime}$ and leads in the closed subshell case to the usual RPA interactions. For this reason, we denote our approximation the "graphical RPA rule" and use it to define the RPA in the open-shell case. Physically, the approximation implies that when the correlated ground state functions $\phi_{a}$ and $\phi_{b}$ interact with the $n_{0} l_{0}^{q-2}$ core as in Figs. 4 and 6, there is no exchange of orbital or spin angular momentum. Furthermore, Brigg's weight factor $\frac{1}{2}(q-2)$ for these interactions is replaced by $2\left(2 l_{0}+1\right)$, implying that the Pauli exclusion principle is ignored and all core magnetic quantum numbers are summed over. The increase in weight factor thus tends to compensate for the inclusion of only zero angular-momentum exchanges.

The graphical RPA rule is shown in Fig. 9. At the top of the figure we show the general structure of the interaction diagrams in Figs. 4 and 6 as well as the weight factor $\frac{1}{2}(q-2)$ and the summation over the quantum numbers $L^{\prime \prime} S$ " of the $n_{0} l_{0}^{q-3}$ configuration. The block labeled $\beta$ indicates an interaction of one of the $n_{0} l_{0}$ electrons with the electron represented by $\phi_{a}$ (cf. Figs. 4 and 6). The middle part of Fig. 9 shows an exact expansion in terms of the orbital and spin angular momenta $\lambda^{\prime}$ and $\sigma^{\prime}$ exchanged between the core $n_{0} l_{0}^{q-2}$ and the interaction block $\beta$. The expansion is an example of the well-known graphical summation rule. ${ }^{22}$ Upon keeping only the term in the summation having $\lambda^{\prime}=\sigma^{\prime}=0$, the summation over $L^{\prime \prime} S^{\prime \prime}$ may be performed analytically. Replacing $\frac{1}{2}(q-2)$ by $2\left(2 l_{0}+1\right)$, we obtain finally the simpler diagram at the bottom of Fig. 9, which is our approximation to the interaction diagram at the top of Fig. 9.

\section{F. Reduction of the diagrams to algebraic expressions}

We summarize here the steps needed to obtain detailed algebraic expressions for each of the terms in Eq. (4) for the first-order transition matrix using graphical methods.

(i) Draw the graphical expression for the firsttransition matrix on the left-hand side of Fig. 4, and for each of the interactions on the right-hand side of Eq. (4) using initial and final states having the forms in Eqs. (5) and (6). For this purpose the rules $(i)-(i x)$ given in Sec. 6.1 of Briggs's review ${ }^{20}$ are used taking account of the necessary modifications, indicated in Secs. III B. and III C. above, needed because the $N$ th electron coordinate is not integrated over. The resulting graphs for the firstorder transition matrix and for the major electronic interactions are shown in Figs. 1, 3, 4, 5, and 6.

(ii) Give each diagram a standard phase by applying the following two modifications of rules (iii) and (iv) from Sec. 4.2 of Briggs's review ${ }^{20}$ :

(a) Reverse the direction of the arrows on all lines which appear as "2nd coupled" at each node of the diagram of the left-hand wave function and as "1st coupled" at each node of the diagram of the right-hand wave function. Note that if a given line whose arrow is reversed is also part of a Coulomb interaction diagram (cf. Fig. 2), then its continuation through the node with the $k$ line also has its arrow reversed.

(b) Reverse the signs on all nodes in each diagram.

(iii) Use our graphical rule shown in Fig. 8 to factor out the magnetic quantum number dependence of each of the diagrams.

(iv) Use our graphical RPA rule shown in Fig. 9 to give an approximate simplified form for the diagrams of the type shown in Figs. 4 and 6 involving interactions of the excited electrons $\phi_{a}$ and $\phi_{b}$ [cf. Eq. (6)] with the ionic core.

(v) Separate each diagram into a spin angularmomentum diagram and an orbital angularmomentum diagram [cf. rule (i), Sec. 4.2 of Briggs's review $\left.^{20}\right]$. Note that the Coulomb interaction, being spin independent, appears only in the orbital diagram; in the spin diagram the $k$ line (cf. Fig. 2) and its associated nodes do not appear.

(vi) Multiply each diagram by the factor $[X]^{1 / 2} \equiv(2 X+1)^{1 / 2}$ for each thick line having angular momentum $X$.

(vii) Each diagram is now in standard form and may be manipulated to obtain an algebraic expres- 
sion for the angular factor it represents by means of standard graphical rules as set out, e.g., by Jucys et al. ${ }^{21}$

\section{THE OPEN-SHELL RPA EQUATIONS}

Section III has presented graphical techniques for the evaluation of individual terms in the equation of motion [Eq. (4)]. One obtains thereby, a single rather complex equation involving the unknown excited electron radial functions $\psi_{\left(\tilde{L}^{\prime} \tilde{S}^{\prime}\right) \in l^{\prime}}^{\alpha}(r), \phi_{a}(r)$, and $\phi_{b}(r)$. In this section we reduce this complex single equation to a set of cou- pled differential equations for the individual finalstate radial functions $\psi_{\left(\tilde{L}^{\prime} \tilde{S}^{\prime}\right) \epsilon l^{\prime}}^{\alpha}(r)$ and for certain linear combinations of the virtually excited electron radial functions $\phi_{a}(r)$ and $\phi_{b}(r)$.

\section{A. Separation of final-state and initial-state correlations}

The magnetic-quantum-number dependence of each of the interactions in Eq. (4) has been extracted analytically by means of the rule, shown in Fig. 8 , which involves an expansion over orbital and

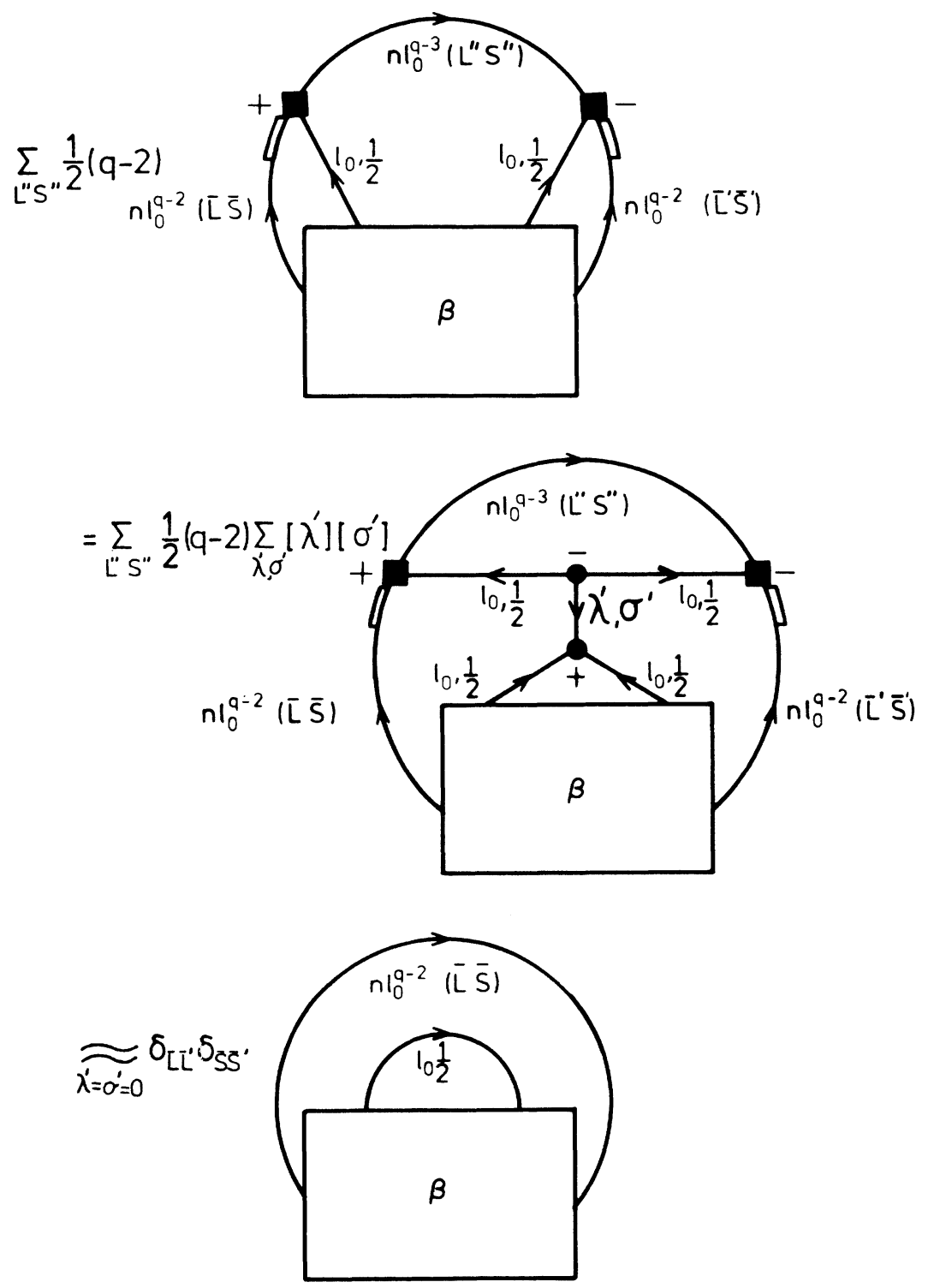

FIG. 9. The graphical RPA rule. See discussion in text. 
spin angular momenta $\lambda$ and $\sigma$. We may single out of Eq. (4) particular values $\lambda^{\prime}$ and $\sigma^{\prime}$ for these quantum numbers and eliminate the magneticquantum-number dependence altogether by first multiplying each term in Eq. (4) by the product,

$$
\left(\begin{array}{ccc}
L_{i} & \lambda^{\prime} & L_{f} \\
\boldsymbol{M}_{i} & m_{\lambda^{\prime}} & -\boldsymbol{M}_{f}
\end{array}\right)\left(\begin{array}{ccc}
S_{i} & \sigma^{\prime} & S_{f} \\
\boldsymbol{M}_{S_{i}} & m_{\sigma^{\prime}} & -\boldsymbol{M}_{S_{f}}
\end{array}\right),
$$

summing over $M_{i}, M_{f}, M_{S_{i}}$, and $M_{S_{f}}$, and making use of the orthogonality of the $3 j$ coefficients. ${ }^{24}$ For each interaction in Eq. (4), we are left then with a closed diagram multiplied by the following factors (cf. Fig. 8): For the final-state interactions, those shown in Figs. 1(a) and 3(a) have the factor

$$
\left(l, \hat{r}_{N}^{\prime}\left|W_{m_{\lambda^{\prime} \sigma_{\sigma^{\prime}}}^{\prime}}^{\lambda^{\prime} \sigma^{\prime}}\right| l_{0}, \hat{r}_{N}\right) P_{n_{0} l_{0}}\left(r_{N}\right) \psi_{(\tilde{L} \widetilde{S}) \epsilon l^{\prime}}^{\alpha}\left(r_{N}^{\prime}\right)
$$

while those shown in Figs. 3(b) and 4 have the factor

$$
\left(l, \widehat{r}_{N}\left|W_{m_{\lambda^{\prime} m_{\sigma^{\prime}}}^{\lambda^{\prime} \sigma^{\prime}}}^{\lambda_{0}}\right| l_{0}, \hat{r}_{N}\right) P_{n_{0} l_{0}}\left(r_{N}\right) P_{n_{0} 1_{0}}\left(r_{N}^{\prime}\right)
$$

for the initial-state interactions, those shown in Figs. 1(b), 6(a), and 6(c) have the factor

$$
\left(l_{0}, \hat{r}_{N}\left|W_{m_{\lambda^{\prime}} \boldsymbol{m}_{\sigma^{\prime}}}^{\lambda^{\prime} \sigma^{\prime}}\right| l, \hat{r}_{N}\right) \phi_{a}\left(r_{N}\right) P_{n_{0} l_{0}}\left(r_{N}^{\prime}\right),
$$

while those shown in Figs. 5, 6(b), and 6(d) have the factor

$$
\left(l_{0}, \widehat{r}_{N}^{\prime}\left|W_{m_{\lambda^{\prime}} \boldsymbol{m}_{\sigma^{\prime}}}^{\lambda^{\prime} \sigma^{\prime}}\right| l, \widehat{r}_{N}\right) P_{n_{0} l_{0}}\left(r_{N}\right) P_{n_{0} l_{0}}\left(r_{N}^{\prime}\right) .
$$

In Eqs. (10) and (11) the double tensor operators $W_{m_{\lambda^{\prime}} m_{\sigma^{\prime}}}^{\lambda^{\prime} \sigma^{\prime}}$ have been defined in Eq. (9) and the wave functions with coordinates $r_{N}$ and $r_{N}^{\prime}$ describe the last electron in the initial and in the final states, whose coordinates are not integrated over. Noting that the double tensors

$$
\left(l, \widehat{r}_{N}^{\prime}\left|W_{m_{\lambda^{\prime} m_{\sigma^{\prime}}}^{\lambda^{\prime} \sigma^{\prime}}}^{\lambda^{\prime}}\right| l_{0}, \hat{r}_{N}\right)
$$

and

$$
\left(l_{0}, \hat{r}_{N}^{\prime}\left|W_{m_{\lambda^{\prime} m_{\sigma^{\prime}}}^{\lambda^{\prime} \sigma^{\prime}}}^{\lambda^{\prime}}\right| l, \hat{r}_{N}\right)
$$

appearing in (10) and (11), respectively, are linearly independent, we may set their coefficients separately equal to zero. Thereby, one obtains a single equation for final-state interactions and a single equation for initial-state interactions. The finalstate interaction equation has the overall factor $P_{n_{0} l_{0}}\left(r_{N}\right)$ and the initial-state interaction equation has the overall factor $P_{n_{0} l_{0}}\left(r_{N}^{\prime}\right)$. Multiplying these equations by $P_{n_{0} l_{0}}\left(r_{N}\right)$ and $P_{n_{0} l_{0}}\left(r_{N}^{\prime}\right)$, respectively, and integrating over $r_{N}$ and $r_{N}^{\prime}$, respectively, replaces these overall factors by unity. In the case-not considered here-where excitations are permitted from several subshells, this latter procedure would give separate equations for each subshell.

\section{B. Definition of new virtual excited orbital functions}

We have thus far reduced Eq. (4) to two coupled equations, with particular values of $\lambda^{\prime}$ and $\sigma^{\prime}$ and no magnetic-quantum-number dependence, which describe, respectively, final-state and initial-state correlations. These two equations are still quite complicated. Careful examination shows, however, that in each term involving the virtually excited electron radial functions $\phi_{a}$ and $\phi_{b}$ the following rather complex summation may be identified:

$$
\begin{aligned}
\Phi(r) \equiv \sum_{\bar{L} \bar{S}} \sum_{L_{p} S_{p}} \sum_{\tilde{L}^{\prime} \tilde{S}^{\prime}} \sum_{l^{\prime}} & \left.b\left(\bar{L} \bar{S}, L_{p} S_{p}, l_{\phi}\right)\left(l_{0}^{q-2} \bar{L} \bar{S} \mid\right\} l_{0}^{q-1} \widetilde{L}^{\prime} \widetilde{S}^{\prime}\right) \\
& \times(q-1)^{1 / 2}\left(\left[L_{i}\right]\left[S_{i}\right]\left[L_{f}\right]\left[S_{f}\right]\left[L_{p}\right]\left[S_{p}\right]\left[\tilde{L}^{\prime}\right]\left[\widetilde{S}^{\prime}\right]\right)^{1 / 2} \mathscr{L} \mathscr{S} \\
& \times\left[\phi_{a}(r)\left(\phi_{b} \mid \psi_{\left(\tilde{L}^{\prime}\right.} \tilde{S}^{\prime}\right) \epsilon l^{\prime}\right. \\
&
\end{aligned}
$$

where $\mathscr{L}$ and $\mathscr{S}$ represent the orbital and spin angular-momentum graphs shown in Fig. 10. $\mathscr{L}$ and $\mathscr{S}$ may be written algebraically in a number of ways, depending upon the choice of dummy summation indices (which we have labeled $\Lambda$ and $\Sigma$ ), one of which is 


$$
\begin{aligned}
& \mathscr{L} \equiv(-1)^{L_{i}+L_{p}+l^{\prime}+\bar{L}+L_{f}+\tilde{L}^{\prime}} \sum_{\Lambda}[\Lambda]\left\{\begin{array}{ccc}
L_{i} & L_{f} & \lambda^{\prime} \\
l_{0} & l_{\phi} & \Lambda
\end{array}\right\}\left\{\begin{array}{ccc}
L_{p} & l_{\phi} & l^{\prime} \\
\Lambda & \bar{L} & L_{i}
\end{array}\right\}\left\{\begin{array}{ccc}
\bar{L} & \Lambda & l^{\prime} \\
L_{f} & \widetilde{L^{\prime}} & l_{0}
\end{array}\right\}, \\
& \mathscr{S} \equiv(-1)^{S_{i}+S_{p}+1 / 2+\bar{S}+S_{f}+\tilde{S}^{\prime}} \sum_{\Sigma}[\Sigma]\left\{\begin{array}{ccc}
S_{i} & S_{f} & \sigma^{\prime} \\
\frac{1}{2} & \frac{1}{2} & \Sigma
\end{array}\right\}\left\{\begin{array}{ccc}
S_{p} & \frac{1}{2} & \frac{1}{2} \\
\Sigma & \bar{S} & S_{i}
\end{array}\right\}\left\{\begin{array}{ccc}
\bar{S} & \Sigma & \frac{1}{2} \\
S_{f} & \widetilde{S}^{\prime} & \frac{1}{2}
\end{array}\right\} .
\end{aligned}
$$

Equation (12) shows firstly that only the particular linear combination of $\phi_{a}$ and $\phi_{b}$ shown in brackets in Eq. (12a) appears in our equations. Secondly, many of the angular factors can be included together with this linear combination of $\phi_{a}$ and $\phi_{b}$ to define new virtually excited orbital functions satisfying a less complicated differential equation. To this end we rewrite Eq. (12a) as

$$
\Phi(r) \equiv-\left(\frac{\left[L_{i}\right]\left[S_{i}\right]\left[L_{f}\right]\left[S_{f}\right]}{\left[l_{\phi}\right]\left[\frac{1}{2}\right]}\right)^{1 / 2} \sum_{\Lambda} \sum_{\Sigma}[\Lambda][\Sigma]\left\{\begin{array}{ccc}
L_{i} & L_{f} & \lambda^{\prime} \\
l_{0} & l_{\phi} & \Lambda
\end{array}\right\}\left\{\begin{array}{ccc}
S_{i} & S_{f} & \sigma^{\prime} \\
\frac{1}{2} & \frac{1}{2} & \Sigma
\end{array}\right\} \phi_{(\Lambda, \Sigma) l_{\phi}}(r)
$$

where

$$
\begin{aligned}
&\left.\phi_{(\Lambda \Sigma) l_{\phi}}(r) \equiv \sum_{\tilde{L} \widetilde{S} l} \sum_{\tilde{L} \bar{S}} \sum_{L_{p} S_{p}} b\left(\bar{L} \bar{S}, L_{p} S_{p}, l_{\phi}\right)\left(l_{0}^{q-2} \bar{L} \bar{S} \mid\right\} l_{0}^{q-1} \tilde{L} \widetilde{S}\right)\left\{(q-1)\left[l_{\phi}\right]\left[\frac{1}{2}\right][\tilde{L}][\widetilde{S}]\left[L_{p}\right]\left[S_{p}\right]\right\}^{1 / 2} \\
& \times(-1)^{1+L_{i}+L_{p}+\bar{L}+\widetilde{L}+l_{\phi}+L_{f}}\left\{\begin{array}{ccc}
L_{p} & l_{\phi} & l_{\phi} \\
\Lambda & \bar{L} & L_{i}
\end{array}\right\}\left\{\begin{array}{ccc}
\bar{L} & \Lambda & l_{\phi} \\
L_{f} & \widetilde{L} & l_{0}
\end{array}\right\} \\
& \\
& \times(-1)^{S_{i}+S_{p}+\bar{S}+\tilde{S}+1 / 2+S_{f}}\left\{\begin{array}{ccc}
S_{p} & \frac{1}{2} & \frac{1}{2} \\
\Sigma & \bar{S} & S_{i}
\end{array}\right\}\left\{\begin{array}{ccc}
\bar{S} & \Sigma & \frac{1}{2} \\
S_{f} & \widetilde{S} & \frac{1}{2}
\end{array}\right\} \\
& \times\left[\phi_{a}(r)\left(\phi_{b} \mid \psi_{(\widetilde{L} \widetilde{S}) \epsilon l}\right)+(-1)^{L_{p}+S_{p}} \phi_{b}(r)\left(\phi_{a} \mid \psi_{(\widetilde{L} \tilde{S}) \epsilon l}\right)\right]
\end{aligned}
$$

Note that we have introduced a factor $-\left[l_{\phi}\right]^{1 / 2}[1 / 2]^{1 / 2}$ in Eq. (13b) and simultaneously divided Eq. (13a) by this factor in order that $\phi_{(\Lambda \Sigma) l_{\phi}}(r)$ reduce exactly in the case of closed-shell atoms to the correlation function $\phi(r)$ defined in Ref. 14. Note also that the allowed values of the summation indices $\Lambda$ and $\Sigma$ are determined from the properties of the $6 j$ coefficients in Eqs. (13a) and (13b) to be given by the triangular delta functions $\left\{l_{0} L_{f} \Lambda\right\},\left\{L_{i} l_{\phi} \Lambda\right\},\left\{\bar{L} l_{\phi} \Lambda\right\},\left\{\frac{1}{2} S_{f} \Sigma\right\}$, $\left\{\frac{1}{2} S_{i} \Sigma\right\}$, and $\left\{\frac{1}{2} \bar{S} \Sigma\right\}$, where each triangular delta function $\{a b c\}$ implies

$$
|a-b| \leq c \leq a+b \text {. }
$$

\section{The coupled equations for individual $\psi_{(\widetilde{L} \widetilde{S}) \epsilon l}(r)$ and $\phi_{(\Lambda \Sigma) l_{\phi}}(r)$ functions}

We still have two differential equations, one describing final-state interactions and the other describing initial-state interactions, each of which now involves the many unknown radial functions $\psi_{(\tilde{L} \widetilde{S}) \epsilon l}(r)$ and $\phi_{(\Lambda \Sigma) l_{\phi}}(r)$ which we must obtain. A set of coupled differential equations for each of these individual unknown radial functions may be obtained by using a well-known orthogonality relation $^{25}$ for the $6 j$ coefficients. This relation serves to project out of our final- and initial-state equations a set of equations for individual values of $L$, $S$, and $\Lambda, \Sigma$, respectively. For this purpose, then, we multiply our final-state interaction equation by

$$
\left[\lambda^{\prime}\right]\left[\sigma^{\prime}\right]\left\{\begin{array}{ccc}
L_{i} & L_{f} & \lambda^{\prime} \\
l & l_{0} & \tilde{L}
\end{array}\right\}\left\{\begin{array}{ccc}
S_{i} & S_{f} & \sigma^{\prime} \\
\frac{1}{2} & \frac{1}{2} & \widetilde{S}
\end{array}\right\},
$$

and sum over $\lambda^{\prime}$ and $\sigma^{\prime}$; we multiply our initialstate interaction equation by 


$$
\left[\lambda^{\prime}\right]\left[\sigma^{\prime}\right]\left\{\begin{array}{lll}
L_{i} & L_{f} & \lambda^{\prime} \\
l_{0} & l_{\phi} & \Lambda
\end{array}\right\}\left\{\begin{array}{ccc}
S_{i} & S_{f} & \sigma^{\prime} \\
\frac{1}{2} & \frac{1}{2} & \Sigma
\end{array}\right\}
$$

and sum over $\lambda^{\prime}$ and $\sigma^{\prime}$. Simplifying the results of this procedure we obtain the following set of coupled equations for the individual radial functions:

$\left(-\frac{1}{2} \frac{d^{2}}{d r^{2}}-\frac{Z}{r}+\frac{1}{2} \frac{l(l+1)}{r^{2}}-\epsilon_{n_{0} l_{0}}-\hbar \omega\right) \psi_{(\tilde{L} \tilde{S}) \epsilon l}^{\alpha}(r)$

$$
\begin{aligned}
& +\sum_{n_{1} l_{1}}^{\neq n_{0} l_{0}} \sum_{k} \mid\left(l \| V_{k}\left(P_{n_{1} l_{1}}, P_{n_{1} l_{1}} ; r\right)|| l\right) \frac{2\left[l_{1}\right]^{1 / 2}}{[l]^{1 / 2}} \delta_{k 0} \psi_{(\widetilde{L} \tilde{S}) \epsilon l}^{\alpha}(r) \\
& \left.+(-1)^{1+k}\left(l|| V^{k}\left(P_{n_{1} l_{1}}, \psi_{(\tilde{L} \tilde{S}) \epsilon l}^{\alpha} ; r\right) \| l_{1}\right) \frac{1}{[l]} P_{n_{1} l_{1}}(r)\right) \\
& +\sum_{\tilde{L}^{\prime} \tilde{S}^{\prime} l^{\prime}} \sum_{k}\left[A\left(\tilde{L}^{\prime},{\widetilde{S^{\prime}}}^{\prime}, l^{\prime}, k\right)\left(l|| V^{k}\left(P_{n_{0} l_{0}}, P_{n_{0} l_{0}} ; r\right)|| l^{\prime}\right) \psi_{\left(\tilde{L}^{\prime} \tilde{S}^{\prime}\right) \in l^{\prime}}^{\alpha}(r)\right. \\
& \left.+B\left(\widetilde{L^{\prime}},{\widetilde{S^{\prime}}}^{\prime}, l^{\prime}, k\right)\left(l\left\|V^{k}\left(P_{n_{0} l_{0}}, \psi_{\left(\tilde{L}^{\prime} \tilde{S}^{\prime}\right) \epsilon l^{\prime}}^{\alpha} ; r\right)\right\| l_{0}\right) P_{n_{0} l_{0}}(r)\right] \\
& +\sum_{\Lambda \Sigma l_{\phi}} \sum_{k} C\left(\Lambda, \Sigma, l_{\phi}, k\right)\left(l\left\|V^{k}\left(\phi_{(\Lambda \Sigma) l_{\phi}}, P_{n_{0} l_{0}} ; r\right)\right\| l_{0}\right) P_{n_{0} l_{0}}(r)=0,
\end{aligned}
$$

where

$$
\begin{aligned}
& A\left(\widetilde{L^{\prime}}, \widetilde{S}^{\prime}, l^{\prime}, k\right) \equiv(-1)^{\tilde{L}+\tilde{L}^{\prime}+L_{f}+l_{0}+l}[\tilde{L}]^{1 / 2}\left[\widetilde{L}^{\prime}\right]^{1 / 2}(q-1) \delta_{\widetilde{S}, \tilde{S}^{\prime}} \\
& \times \sum_{\bar{L} \bar{S}}(-1)^{\bar{L}}\left(l_{0}^{q-1} \tilde{L} \widetilde{\boldsymbol{S}}\left\{\mid l_{0}^{q-2} \bar{L} \overline{\boldsymbol{S}}\right)\left(l_{0}^{q-2} \bar{L} \overline{\boldsymbol{S}} \mid\right\} l_{0}^{q-1} \widetilde{L^{\prime}}{\widetilde{S^{\prime}}}^{\prime}\right)\left\{\begin{array}{ccc}
l_{0} & \tilde{L} & \bar{L} \\
\widetilde{L^{\prime}} & l_{0} & k
\end{array}\right\}\left\{\begin{array}{ccc}
L_{f} & l^{\prime} & \widetilde{L^{\prime}} \\
k & \widetilde{L} & l
\end{array}\right\},
\end{aligned}
$$

$B\left(\widetilde{L^{\prime}}, \widetilde{S^{\prime}}, l^{\prime}, k\right) \equiv(-1)^{l_{0}+l^{\prime}-\widetilde{S}-\widetilde{S}^{\prime}}(q-1)\left([\widetilde{L}]\left[\widetilde{L^{\prime}}\right][\widetilde{S}]\left[\widetilde{S^{\prime}}\right]\right)^{1 / 2}$

$$
\times \sum_{\bar{L} \bar{S}}\left(l_{0}^{q-1} \widetilde{L} \widetilde{S}\left\{\mid l_{0}^{q-2} \bar{L} \bar{S}\right)\left(l_{0}^{q-2} \bar{L} \bar{S} \mid\right\} l_{0}^{q-1} \widetilde{L}^{\prime} \widetilde{S}^{\prime}\right)\left\{\begin{array}{ccc}
\bar{L} & l_{0} & \widetilde{L} \\
l_{0} & k & l \\
\widetilde{L}^{\prime} & l^{\prime} & L_{f}
\end{array}\right\}\left\{\begin{array}{ccc}
\widetilde{S} & S_{f} & \frac{1}{2} \\
\widetilde{S^{\prime}} & \bar{S} & \frac{1}{2}
\end{array}\right\},
$$

$$
\begin{aligned}
C\left(\Lambda, \Sigma, l_{\phi}, k\right) \equiv & \frac{(-1)^{L_{f}+S_{f}-\tilde{L}-\tilde{S}-l+1 / 2}[\tilde{L}][\widetilde{S}][\Lambda][\Sigma]}{\left(l_{0}^{q}\left\{\mid l_{0}^{q-1} \widetilde{L} \widetilde{S}\right)\left(q\left[l_{\phi}\right][1 / 2]\right)^{1 / 2}\right.} \\
& \times(1-1)^{k+\widetilde{S}-\Sigma} \frac{\delta_{S_{i} S_{f}}}{\left[S_{i}\right]}\left\{\begin{array}{ccc}
L_{i} & L_{f} & k \\
l & l_{0} & \tilde{L}
\end{array}\right\}\left\{\begin{array}{lll}
L_{i} & L_{f} & k \\
l_{0} & l_{\phi} & \Lambda
\end{array}\right\} \\
& \left.+(-1)^{1+l_{0}+l_{\phi}+L_{i}+L_{f}+\tilde{L}+\Lambda} \frac{\delta_{\tilde{S} \Sigma}}{[\widetilde{S}]}\left\{S_{f} \frac{1}{2} \widetilde{S}\right\}\left\{\begin{array}{ccc}
k & \Lambda & \tilde{L} \\
L_{i} & l_{0} & l_{\phi}
\end{array}\right\}\left\{\begin{array}{ccc}
k & \Lambda & \tilde{L} \\
L_{f} & l & l_{0}
\end{array}\right\}\right),
\end{aligned}
$$




$$
\begin{aligned}
& \left(-\frac{1}{2} \frac{d^{2}}{d r^{2}}-\frac{Z}{r}+\frac{1}{2} \frac{l_{\phi}\left(l_{\phi}+1\right)}{r^{2}}+\hbar \omega-\epsilon_{n_{0} l_{0}}\right) \phi_{(\Lambda \Sigma) l_{\phi}}(r) \\
& +\sum_{n_{1} l_{1}}^{\neq n_{0} l_{0}} \sum_{k} \int\left(l_{\phi}\left\|V^{k}\left(P_{n_{1} l_{1}}, P_{n_{1} l_{1}} ; r\right)\right\| l_{\phi}\right) \delta_{k 0} \frac{2\left[l_{1}\right]^{1 / 2}}{\left[l_{\phi}\right]^{1 / 2}} \phi_{(\Lambda \Sigma) l_{\phi}}(r) \\
& \left.+(-1)^{1+k}\left(l_{1} \| V^{k}\left(\phi_{(\Lambda \Sigma) l_{\phi}}, P_{n_{1} l_{1}} ; r\right)|| l_{\phi}\right) \frac{1}{\left[l_{\phi}\right]} P_{n_{1} l_{1}}(r)\right) \\
& +\sum_{\Lambda^{\prime} \Sigma^{\prime} l_{\phi}^{\prime}} \sum_{k}\left[D\left(\Lambda^{\prime}, \Sigma^{\prime}, l_{\phi}^{\prime}, k\right)\left(l_{\phi}^{\prime} \| V^{k}\left(P_{n_{0} l_{0}}, P_{n_{0} l_{0}} ; r\right)|| l_{\phi}\right) \phi_{\left(\Lambda^{\prime} \Sigma^{\prime}\right) l_{\phi}^{\prime}}(r)\right. \\
& \left.+E\left(\Lambda^{\prime}, \Sigma^{\prime}, l_{\phi}^{\prime}, k\right)\left(l_{0} \| V^{k}\left(\phi_{\left(\Lambda^{\prime} \Sigma^{\prime}\right) l_{\phi}^{\prime}}, P_{n_{0} l_{0}} ; r\right)|| l_{\phi}\right) P_{n_{0} l_{0}}(r)\right] \\
& +\sum_{\tilde{L}^{\prime} \tilde{S}^{\prime} l^{\prime}} \sum_{k} F\left(\widetilde{L}^{\prime}, \widetilde{S}^{\prime}, l^{\prime}, k\right)\left(l_{0}\left\|V^{k}\left(P_{n_{0} l_{0}}, \psi_{\left(\tilde{L}^{\prime} \tilde{S}^{\prime}\right) \epsilon l^{\prime}}^{\alpha} ; r\right)\right\| l_{\phi}\right) P_{n_{0} l_{0}}(r)=0,
\end{aligned}
$$

where

$$
\begin{aligned}
& D\left(\Lambda^{\prime}, \Sigma^{\prime}, l_{\phi}^{\prime}, k\right) \equiv \delta_{\Lambda \Lambda^{\prime}} \delta_{\Sigma \Sigma^{\prime}} \delta_{l_{\phi} l_{\phi}^{\prime}} \delta_{k 0} \frac{2\left[l_{0}\right]^{1 / 2}}{\left[l_{\phi}^{\prime}\right]^{1 / 2}} \\
& +(-1)^{1+l_{0}+l_{\phi}+L_{i}+L_{f}+\Lambda+\Lambda^{\prime}} \delta_{\Sigma \Sigma^{\prime}} \frac{\left[\Lambda^{\prime}\right]\left[l_{\phi}\right]^{1 / 2}}{\left[l_{\phi}^{\prime}\right]^{1 / 2}}\left\{\begin{array}{ccc}
k & \Lambda & \Lambda^{\prime} \\
L_{i} & l_{\phi}^{\prime} & l_{\phi}
\end{array}\right\}\left\{\begin{array}{ccc}
k & \Lambda & \Lambda^{\prime} \\
L_{f} & l_{0} & l_{0}
\end{array}\right\} \text {, }
\end{aligned}
$$

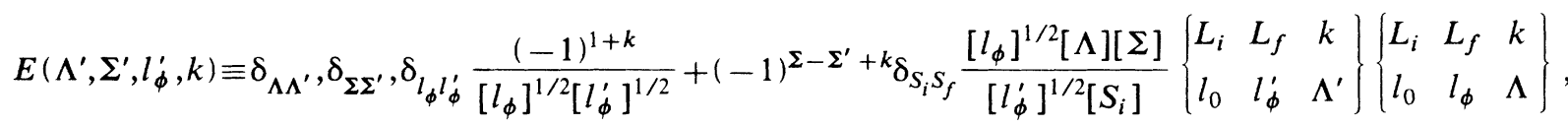

$$
\begin{aligned}
& F\left(\widetilde{L^{\prime},} \widetilde{S^{\prime}}, l^{\prime}, k\right) \equiv(-1)^{k+l_{\phi}+L_{f}+\widetilde{L}^{\prime}+1 / 2+\widetilde{S^{\prime}}+S_{f}-\Sigma}(q-1)\left(q\left[l_{\phi}\right]\left[\frac{1}{2}\right]\left[\widetilde{L^{\prime}}\right]\left[\widetilde{S^{\prime}}\right]\right)^{1 / 2} \\
& \times \sum_{\tilde{L} \bar{S}} \sum_{\widetilde{L} \widetilde{S}}(-1)^{-\widetilde{S}}[\widetilde{L}]^{1 / 2}[\widetilde{S}]^{1 / 2}\left(l_{0}^{q} L_{i} S_{i}\left\{\mid l_{0}^{q-1} \widetilde{L} \widetilde{S}\right)\left(l_{0}^{q-1} \widetilde{L} \widetilde{S}\left\{\mid l_{0}^{q-2} \bar{L} \bar{S}\right)\left(l_{0}^{q-2} \bar{L} \bar{S} \mid\right\} l_{0}^{q-1} \widetilde{L}^{\prime} \widetilde{S}^{\prime}\right)\right. \\
& \times\left\{\begin{array}{ccc}
\bar{L} & \Lambda & l^{\prime} \\
L_{f} & \tilde{L}^{\prime} & l_{0}
\end{array}\right\}\left\{\begin{array}{lll}
\bar{L} & l_{0} & \tilde{L} \\
l^{\prime} & k & l_{0} \\
\Lambda & l_{\phi} & L_{i}
\end{array}\right\}\left\{\begin{array}{ccc}
\bar{S} & \Sigma & \frac{1}{2} \\
S_{f} & \widetilde{S^{\prime}} & \frac{1}{2}
\end{array}\right\}\left\{\begin{array}{ccc}
\bar{S} & \Sigma & \frac{1}{2} \\
S_{i} & \tilde{S} & \frac{1}{2}
\end{array}\right\} .
\end{aligned}
$$

In Eqs. (14a) and (15a) the one-particle operator terms in the first set of parentheses are calculated from diagrams similar to those in Figs. 1(a) and 1(b), respectively. The terms in the second set of parentheses in Eqs. (14a) and (15a) describe the interaction of an excited electron with the closed subshells $n_{1} l_{1} \neq n_{0} l_{0}$. The final-state interactions in Eq. (14a) having the angular factors in Eqs. (14b), (14c), and (14d) are obtained from the diagrams shown in Figs. 3(a), 3(b), and 4, respectively. The initial-state interactions in Eq. (15a) having the an- gular factors in Eq. (15b) are obtained from the diagrams in Figs. 6(a) and 6(b); those having the angular factors in Eq. (15c) are obtained from the diagrams in Figs. 6(b) and 6(d); and those having the angular factor in Eq. (15d) are obtained from the diagram in Fig. 5. Note that orthogonality of the functions $\psi_{(\tilde{L} \widetilde{S}) \epsilon l}(r)$ and $\phi_{(\mathbf{\Lambda} \Sigma) l_{\phi}}(r)$ to bound orbitals having the same angular momentum is easily imposed by adding certain inter-action terms in Eqs. (14a) and (15a) whose form is dependent on the choice of core orbitals. $^{26}$ 


\section{Special cases of the equations}

In the special case where ground-state correlations are ignored (i.e., setting all $\phi$ functions equal to zero), Eq. (14) reduces to the simplest form of the usual close-coupling equations. For example, in the case of atoms having an open $p$ subshell $p^{q}$, our equations reduce to those of Smith et al. ${ }^{27}$ : The direct interaction potential in their Eq. (19) and the exchange matrix element in their Eq. (22) correspond exactly with the direct and exchange interactions in our Eq. (14a).

In the important special case of closed-shell atoms our equations reduce to the usual RPA equations. One notes that in this case if the excited state is produced by an electric dipole transition then $\widetilde{L}=\widetilde{L}^{\prime}=l_{0}, \widetilde{S}={\widetilde{S^{\prime}}}^{\prime}=\frac{1}{2}, L_{i}=S_{i}=S_{f}=0$, $L_{f}=1, q=4 l_{0}+2$, and also

$$
\left(l_{0}^{4 l_{0}+1}\left\{\mid l_{0}^{4 l_{0}} \bar{L} \bar{S}\right)=\frac{\left(2-(-1)^{\bar{L}}\right)^{1 / 2}[\bar{L}]^{1 / 2}}{\left[l_{0}\right]^{1 / 2}\left(4 l_{0}+1\right)^{1 / 2}} .\right.
$$

The coefficients in Eqs. 14(b), 14(c), 14(d), 15(b), 15(c), and 15(d) become

$$
\begin{aligned}
& A_{\mathrm{cl}}=\frac{2\left[l_{0}\right]^{1 / 2}}{[l]^{1 / 2}} \delta_{l l^{\prime}} \delta_{k 0}-\left\{\begin{array}{ccc}
l_{0} & l_{0} & k \\
l^{\prime} & l & 1
\end{array}\right\}, \\
& B_{\mathrm{cl}}=-\frac{2}{3} \delta_{k 1}+\frac{\delta_{l l^{\prime}}}{[l]}, \\
& C_{\mathrm{cl}}=(-1)^{1+l+l_{\phi}} \delta_{k 1} \frac{2}{3}+(-1)^{k+l_{0}+l_{\phi}}\left\{\begin{array}{lll}
l_{\phi} & l_{0} & k \\
l & l_{0} & 1
\end{array}\right\}, \\
& D_{\mathrm{cl}}=\frac{2\left[l_{0}\right]^{1 / 2}}{\left[l_{\phi}\right]^{1 / 2}} \delta_{l_{\phi} l_{\phi}^{\prime}} \delta_{k 0}+(-1)^{l_{0}+l_{\phi}+k}\left\{\begin{array}{lll}
l_{\phi} & l_{\phi}^{\prime} & k \\
l_{0} & l_{0} & 1
\end{array}\right\},
\end{aligned}
$$

$$
\begin{aligned}
& E_{\mathrm{cl}}=(-1)^{1+l_{\phi}^{\prime}+l_{\phi}} \frac{2}{3} \frac{\left[l_{\phi}\right]}{\left[l_{\phi}^{\prime}\right]} \delta_{k 1} \\
& +(-1)^{1+k} \frac{\delta_{l_{\phi} l_{\phi}^{\prime}}}{\left[l_{\phi}^{\prime}\right]^{1 / 2}\left[l_{\phi}\right]^{1 / 2}}, \\
& F_{\mathrm{cl}}=-\frac{2}{3} \delta_{k 1}+(-1)^{1+k} \sum_{\bar{L}}(-1)^{\bar{L}}[\bar{L}] \\
& \times\left\{\begin{array}{lll}
l_{0} & l_{\phi} & k \\
l^{\prime} & l_{0} & \bar{L}
\end{array}\right\}\left\{\begin{array}{lll}
l_{0} & l_{\phi} & 1 \\
l^{\prime} & l_{0} & \bar{L}
\end{array}\right\},
\end{aligned}
$$
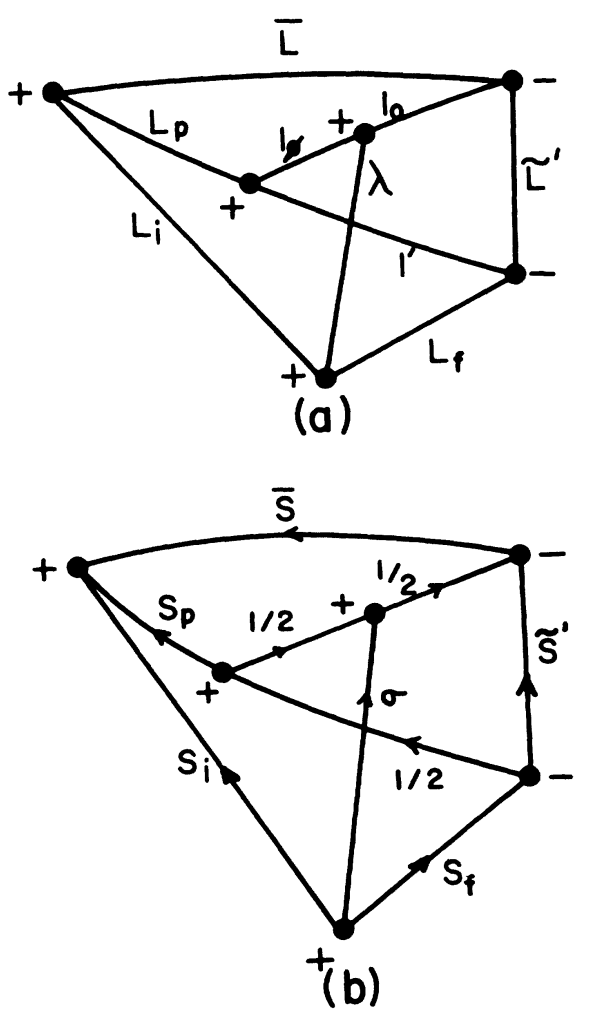

FIG. 10. Graphical representations for the angular factors $\mathscr{L}$ [shown in (a)] and $\mathscr{S}$ [shown in (b)] appearing in Eq. (12).

where the subscript cl denotes closed shells. In the special case of a closed $p$ subshell from which only $d$ excitations are considered in both final and initial states (i.e., $l=l^{\prime}=l_{\phi}=l_{\phi}^{\prime}=2$ ), substitution of Eq. (16) in Eqs. (14a) and (15a) gives exactly Eqs. (21a) and (21b) of Chang and Fano ${ }^{14 b}$ [after substituting their Eqs. (7) and (9)].

\section{ELECTRIC-DIPOLE MATRIX ELEMENTS}

The radial functions $\phi_{(\widetilde{L} \widetilde{S}) \epsilon l}(r)$ and $\phi_{(\Lambda \Sigma) l_{\phi}}(r)$ which are obtained as the solutions of Eqs. (14) and (15) can now be used to compute matrix elements of any one-body operator according to Eq. (2). The graphical procedure for calculating such matrix elements is to insert the graphical representation for the operator (which indicates its angular-momentum properties) into the graphical representation for the first-order transition matrix shown in Fig. 1. The graphical representation of Briggs $^{20}$ for the $q$ th spherical component of the electric-dipole transition operator is shown in Fig. 
11. The double bar on the " 1 " line represents the following reduced dipole matrix element:

$$
\begin{aligned}
& \left\langle n_{1} l_{1}\|r\| n_{2} l_{2}\right\rangle \\
& \quad \equiv(-1)^{l_{1}-l^{\prime}} l_{>}^{1 / 2} \int_{0}^{\infty} P_{n_{1} l_{1}}(r) r P_{n_{2} l_{2}}(r) d r,
\end{aligned}
$$

where

$$
l_{>}=\max \left(l_{1}, l_{2}\right) .
$$

The graph in Fig. 11 is inserted between the gaps (indicating the electron with coordinates $r_{N}, \Omega_{N}$ ) in the diagram for the first-order transition matrix, shown in Figs. 1(a) and 1(b). One may use Briggs's rules $^{20}$ to evaluate the resulting diagram. One obtains for the reduced dipole matrix elements the following expressions:

$$
\begin{aligned}
D_{\epsilon}^{\alpha}= & \sum_{\widetilde{L} \widetilde{S} l} G(\tilde{L}, \widetilde{S}, l)\left\langle P_{n_{0} l_{0}}\|r\| \psi_{(\widetilde{L} \widetilde{S}) \epsilon l}^{\alpha}\right\rangle \\
& +\sum_{\Lambda \Sigma l_{\phi}} H\left(\Lambda, \Sigma, l_{\phi}\right)\left\langle\phi_{(\Lambda \Sigma) l_{\phi}}^{\alpha}\|r\| P_{n_{0} l_{0}}\right\rangle,
\end{aligned}
$$

where

$$
\begin{aligned}
G(\tilde{L}, \widetilde{S}, l) \equiv & \delta_{S_{i} S_{f}}(-1)^{L_{f}+\tilde{L}+l+1} q^{1 / 2}\left(l_{0}^{q}\left\{\mid l_{0}^{q-1} \tilde{L} \widetilde{S}\right)\right. \\
& \times\left[L_{i}\right]^{1 / 2}\left[L_{f}\right]^{1 / 2}\left\{\begin{array}{ccc}
l_{0} & L_{i} & \tilde{L} \\
L_{f} & l & 1
\end{array}\right\}
\end{aligned}
$$

and

$$
\begin{aligned}
H\left(\Lambda, \Sigma, l_{\phi}\right) \equiv & \delta_{S_{i} S_{f}}(-1)^{\Sigma+1 / 2-S_{i}}\left\{S_{i} \frac{1}{2} \Sigma\right\} \\
& \times\left(\frac{\left[L_{i}\right]\left[L_{f}\right]}{\left[l_{\phi}\right]\left[\frac{1}{2}\right]}\right)^{1 / 2}[\Lambda][\Sigma]\left\{\begin{array}{lll}
L_{i} & L_{f} & 1 \\
l_{0} & l_{\phi} & \Lambda
\end{array}\right\}
\end{aligned}
$$

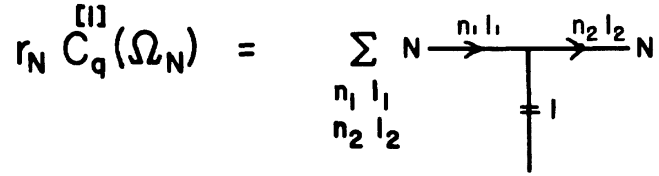

FIG. 11. Brigg's graphical representation of the $q$ th spherical component of the electric-dipole operator.

In Eq. (18c) the symbol $\left\{S_{i} \frac{1}{2} \Sigma\right\}$ represents the socalled triangular delta function, which is unity if the relation, $\left|S_{i}-\frac{1}{2}\right| \leq \Sigma \leq S_{i}+\frac{1}{2}$, is satisfied and zero otherwise. All measureable quantities related to single-electron, electric-dipole transitions such as absorption intensities and photoelectron angular distributions may be calculated theoretically from the dipole amplitudes $D_{\epsilon}^{\alpha}$.

\section{ACKNOWLEDGMENTS}

A.F.S. wishes to acknowledge the hospitality of the Fakultät für Physik der Universität Freiburg where this work was completed and where he was supported in part by an Alexander von Humboldt Research Fellowship. We also acknowledge the support of the U.S. Department of Energy under Contract No. EY-76-S-02-2892. We also thank Professor T. N. Chang and Professor U. Fano for discussions concerning their own work on RPA equations for closed-shell atoms.
*Permanent address.

${ }^{1}$ A. F. Starace, Theory of Atomic Photoionization, Handbuch der Physik, Vol. 31, edited by W. Mehlhorn (Springer, Berlin, 1982).

${ }^{2}$ M. Ya. Amusia and N. A. Cherepkov, Case Stud. At. Phys. 5, 47 (1975).

${ }^{3} \mathrm{G}$. Wendin, Photoionization and Other Probes of Many-Electron Interactions, edited by F. J. Wuilleumier (Plenum, New York, 1976), pp. 61-82.

${ }^{4}$ H. P. Kelly, Photoionization and Other Probes of Many-Electron Interactions, edited by F. J. Wuilleumier (Plenum, New York, 1976), pp. 83-109.
${ }^{5}$ P. G. Burke and W. D. Robb, Adv. At. Mol. Phys. 11, 143 (1975).

${ }^{6}$ P. G. Burke and K. T. Taylor, J. Phys. B $\underline{8}, 2620$ (1975).

${ }^{7}$ J. R. Swanson and L. Armstrong, Jr., Phys. Rev. A $\underline{15}$, 661 (1977); 16, 1117 (1977).

${ }^{8}$ L. Armstrong, Jr., J. Phys. B ㄱ, 2320 (1974).

${ }^{9}$ D. J. Rowe and C. Ngo-Trong, Rev. Mod. Phys. 47, 471 (1975).

${ }^{10}$ E. Dalgaard, J. Phys. B $\underline{8}, 695$ (1975).

${ }^{11}$ A. F. Starace and L. Armstrong, Jr., Phys. Rev. A $\underline{13}$, 1850 (1976). 
${ }^{12}$ N. A. Cherepkov and L. V. Chernysheva, Phys. Lett. 60A, 103 (1977); Izv. Akad. Nauk. SSSR, Ser. Fiz. 41, 2518 (1977) [Bull. Acad. Sci. USSR, Phys. Ser. 41, 47 (1977)].

${ }^{13}$ P. O. Löwdin, Phys. Rev. 97, 1474 (1955).

${ }^{14}$ (a) T. N. Chang and U. Fano, Phys. Rev. A 13 , 263 (1976); (b) 13, 282 (1976).

${ }^{15}$ K. Smith, R. J. W. Henry, and P. G. Burke, Phys. Rev. 147, 21 (1966).

${ }^{16}$ T. N. Chang, Phys. Rev. A $\underline{15}, 2392$ (1977); 16, 1171 (1977); 18, 1448 (1978).

${ }^{17}$ S. Shahabi and A. F. Starace, Bull. Am. Phys. Soc. 23, 1106 (1978).

${ }^{18}$ A. F. Starace and S. Shahabi, Nobel Symposium 46: Many Body Theory of Atomic Systems, Gothenburg, Sweden, June, 1979 [Phys. Scr. 21, 368 (1980)].

${ }^{19}$ The choice of core orbitals in atomic photoionization calculations is not clear cut. The use of neutral atom orbitals for both the initial- and final-state core orbitals (i.e., the frozen-core approximation) is convenient because of its simplicity. However, when relaxation effects are large, the use of relaxed core ionic wave functions may be preferable. This paper is not concerned with the precise characterization of these core orbitals. A discussion of the problem in the context of the random-phase approximation (for closed-shell atoms) has recently been given by M. Ya Amusia in Atomic Physics 5, edited by R. Marrus, M. Prior, and H. Shugart (Plenum, New York, 1977), pp. 537-565.

20J. S. Briggs, Rev. Mod. Phys. 43, 189 (1971).

${ }^{21}$ A. P. Yutsis (Jucys), I. B. Levinson, and V. V. Vanagas, The Theory of Angular Momentum (Israel Program for Scientific Translations, Jerusalem, 1962).

${ }^{22}$ See Ref. 21, pp. 46-7.

${ }^{23}$ P. G. H. Sandars, in Brandeis University Summer Institute in Theoretical Physics, 1969: Atomic Physics and Astrophysics, Vol. 1, edited by M. Chrétien and E. Lipworth (Gordon and Breach, New York, 1971), Sec. 4.3.

${ }^{24}$ See Ref. 21, p. 152.

${ }^{25}$ See Ref. 21, p. 141, Eq. (A.6.1).

${ }^{26} \mathrm{H}$. J. Silverstone and M. L. Yin, J. Chem. Phys. $\underline{49}$, 2026 (1968); S. Huzinaga and C. Arnau, Phys. Rev. A 1, 1285 (1970); see also the brief discussion by H. P. Kelly, in Photoionization and Other Probes of ManyElectron Interactions, edited by F. J. Wuilleumier (Plenum, New York, 1976), pp. 90-91.

${ }^{27}$ See Ref. 15. The notation of Ref. 15 is related to ours as follows: $L_{i} \rightarrow \widetilde{L}, L_{j} \rightarrow \widetilde{L^{\prime}}, l_{i} \rightarrow l, l_{j} \rightarrow l^{\prime}, L \rightarrow L_{f}, S \rightarrow S_{f}$, $q \rightarrow q-1, L_{2} \rightarrow \bar{L}, S_{2} \rightarrow \bar{S}, \lambda \rightarrow k, 1 \rightarrow l_{0}$. 